\title{
Contrast Sensitivity in Diabetic Patients without Retinopathy And It's Correlation with the Duration of Diabetes And Glycemic Control
}

\author{
Dr Rashmi $S^{1}$, Dr. Rejitha Chinnu Varghese ${ }^{2},{ }^{3}$ Dr Anupama B, \\ ${ }^{4}$ Dr Vidya Hegde, ${ }^{5}$ Dr Rashmi Jain, ${ }^{6}$ Himani Kotian \\ ${ }^{1}$ (Ophthalmology, Yenepoya Medical College, India) \\ ${ }^{2}$ (Ophthalmology, Yenepoya Medical College, India)
}

\begin{abstract}
Purpose : To measure the contrast sensitivity in adults with type 2 diabetes mellitus without retinopathy and to compare it with age matched controls and also to correlate the contrast sensitivity in these patients with the duration of diabetes and glycemic control.

Methods: This cross sectional study included 100 diabetic patients and 100 controls. Detailed history, ocular examination, measurement of contrast sensitivity and glycosylated hemoglobin values were measured. Statistical analysis was done using Spearman's coefficient of correlation

Results: Diabetic patients were found to have reduced contrast sensitivity as compared to healthy controls. Also it was found that contrast sensitivity decreased as the duration of diabetes increased and glycemic controls worsened.

Conclusion: Contrast sensitivity could be a useful clinical tool in assessing retinal function in diabetic patients even before any retinopathy changes become visible.
\end{abstract}

Keywords:- Contrast sensitivity, Diabetes Mellitus, Retinopathy

\section{INTRODUCTION}

Diabetes mellitus is one of the most common chronic illnesses. Diabetic retinopathy refers to the retinal changes seen in patients with diabetes mellitus. It is one of the leading causes of blindness. Diabetes affects the normal function of the retinal neurons. Both vascular and metabolic factors play a role in its pathogenesis. Contrast sensitivity is a function of the retina and it evaluates the central vision. It is the ability of the visual system to realise differences between objects and background at finest detail. In a patient with diabetes, contrast sensitivity may be reduced even before the development of diabetic retinopathy and even when the visual acuity is normal. Hence measuring contrast sensitivity may be a useful tool for early detection of abnormalities in retinal functions in diabetics. It may also help in analysing the relationship between metabolic control and retinal function. The purpose of this study was to measure the contrast sensitivity in adults with Type 2 Diabetes Mellitus without retinopathy and to compare it with age matched controls. Another objective of this study was to correlate the contrast sensitivity in these patients with the duration of diabetes and glycemic control.

\section{MATERIALS AND METHODS}

This is a cross sectional study conducted on patients with Type 2 Diabetes Mellitus, without any diabetic retinopathy changes, presenting to the ophthalmology out-patient department of a tertiary eye care hospital. Approval from the institutional ethics committee and written informed consent from participants of this study were obtained. The study included 100 diabetic patients and 100 healthy patients as controls. In Group 1, all adults with Type 2 Diabetes Mellitus who were willing to participate in this study were included. Group 2 included healthy adults as controls. Patients with any other retinal or ocular diseases (infections and inflammations), patients with best corrected visual acuity of less than 6/24, children, elderly, and illiterate, psychiatric and mentally challenged patients were excluded from the study. Age and gender of the patients were noted. A brief ocular and systemic history was taken regarding the purpose of ophthalmology consultation, history of diminution of vision, any other ocular disorders and treatment for the same. Enquiry regarding the duration of diabetes, treatment, and diabetic control was made. Patient's $\mathrm{HbA} 1 \mathrm{c}$ values were measured to determine their glycemic control. Vision in each eye was assessed using Snellen's chart. Contrast sensitivity was measured using Appasamy i chart. It has a grey scale of letter chart and $\mathrm{E}$ chart of font size corresponding to Snellen's test type $6 / 24$. There are lines of decreasing contrast corresponding to $100 \%, 80 \%, 60 \%, 40 \%, 20 \%$, $10 \%, 5 \%, 4 \%$. Normal value of Contrast sensitivity is $5 \%$ and less. Those values above $5 \%$ are abnormal, which indicates a decrease in the Contrast sensitivity. Testing was carried out at a distance of 3 meter (10 feet) with the patient wearing his best spectacle correction. The anterior segment was examined using slit-lamp. The 
posterior segment was examined using $+78 \mathrm{D}$ lens and slit lamp biomicroscopy after dilating the pupils. Detailed retinal examination was done to rule out any evidence of diabetic retinopathy changes. Patients with even one microaneurysm or intraretinal hemorrhage (earliest ophthalmoscopic evidence of diabetic retinopathy) were excluded from the study. Statistical analysis was done using Spearman's coefficient of correlation.

\section{RESULTS}

Frequency distributions of gender and age for cases and controls show that both groups are comparable. (Table 1 and table 2)

Table 1: Frequency distribution of gender for cases and control

\begin{tabular}{|c|c|c|}
\hline & Cases & Control \\
\hline Gender & Frequency & Frequency \\
\hline Female & 54 & 63 \\
\hline Male & 46 & 37 \\
\hline Total & 100 & 100 \\
\hline
\end{tabular}

Table 2: Mean age of cases and controls

\begin{tabular}{|l|r|r|r|}
\hline & \multicolumn{1}{|c|}{ Minimum } & Maximum & \multicolumn{1}{c|}{ Mean } \\
\hline Age(cases) & 40 & 62 & 54.21 \\
Age(controls) & 40 & 64 & 52.28 \\
& & & \\
\hline
\end{tabular}

Contrast sensitivity of cases were compared with that of controls using Spearman's coefficient of correlation. There is a negative correlation between contrast sensitivity of cases as compared to controls (Rho value is -0.025) Hence Contrast sensitivity among diabetic patients is reduced as compared to controls. The contrast sensitivity among diabetic patients was correlated with their duration of diabetes and glycemic control using Spearman's coefficient of correlation. There is a moderate correlation between contrast and duration (Rho value 0.647 ). There is a weak or small correlation between contrast and glycemic control (Rho value 0.441).As the duration increases and glycemic control worsens, there occurred a decrease in contrast sensitivity. (Table 3)

Table 3: Comparison of contrast sensitivity between cases and controls and their correlation with the duration of diabetes and glycemic control.

\begin{tabular}{|l|l|}
\hline & Co efficient of correlation( Rho value) \\
\hline Correlation of contrast ( cases with controls) & -0.025 \\
\hline Correlation of with duration of diabetes & 0.647 \\
\hline Correlation of contrast with glycemic control & 0.441 \\
\hline
\end{tabular}

\section{DISCUSSION}

Several attempts have been made to develop a test predictive of the development of retinopathy. Changes in visual function in diabetics occur before any structural abnormalities can be detected by ophthalmoscopy or even by fluorescein angiography. Contrast sensitivity is a potential tool for screening early stages in diabetic retinopathy. ${ }^{(1)}$

Contrast sensitivity can be a good indicator of the retinal health before retinopathy changes become actually visible. Contrast sensitivity may be impaired even in the presence of a normal visual acuity.

Khosla PK et al evaluated contrast sensitivity in 22 diabetic patients (22 eyes without retinopathy and 16 eyes with background retinopathy on fluorescein angiography) and 10 control subjects (20 eyes) matched for age and sex. Contrast sensitivity was significantly lower in diabetic eyes with retinopathy than in the normal eyes $(p=$ $0.011)$ or the diabetic eyes without retinopathy $(\mathrm{p}=0.033)$. They opine that the test may be of value in screening diabetic patients for retinopathy in primary care facilities. ${ }^{(2)}$

In another study also similar results were seen. Fifteen diabetic patients $(6 / 20$ with retinopathy and $9 / 22$ without) had test scores more than two standard deviations below the norm for age-matched controls. ${ }^{(3)}$

A study was conducted by Verotti A et al to measure contrast sensitivity in diabetics with and without retinopathy and also to analyze the relationship of metabolic control to the presence and severity of retinopathy. 
The results showed an obvious decrease in contrast sensitivity in diabetic retinopathy. Even patients without retinopathy showed reduced contrast sensitivity when compared to controls. Also there was a definite relation between contrast sense and glycemic control. ${ }^{(4)}$

Dosso AA et al evaluated contrast sensitivity in insulin resistant obese individuals and in aretinopathic diabetic patients and found that it was low. This suggests that an early neurosensory dysfunction may occur without any visible changes in the retina. ${ }^{(5)}$

Another study evaluated contrast sensitivity, colour vision and visual acuity in diabetic patients. They found that comparing to the control group, there was a statistically significant loss of contrast sensitivity in the diabetic eyes without retinopathy. ${ }^{(1)}$

A study by DeMarco R et al found that patients with diabetes mellitus with no retinopathy had normal contrast sensitivity and that contrast thresholds were not significantly related to the duration of diabetes and glycemic control. $^{(6)}$

Misra et al also found association between contrast sensitivity and visually acuity as well as glycosylated haemoglobin in patients with Type 2 Diabetes Mellitus. ${ }^{(7)}$

Our study found that there is some reduction in contrast sensitivity in diabetic patients even before they develop visible retinal changes on ophthalmoscopy. However this reduction is not statistically significant when compared with healthy controls. A reduction in contrast sensitivity was associated with duration of diabetes and glycemic control, however it was not statistically significant. We may require a larger sample size for confirmation of the same.

\section{CONCLUSION}

Diabetic patients have reduced contrast sensitivity as compared to healthy controls. As the duration of diabetes increases and glycemic control worsens, these diabetic patients have a decrease in the contrast sensitivity. This observation may be clinically significant even though it was not statistically significant in our study. Hence it can be used clinically as a useful tool in assessing retinal function in diabetic patients even before any retinopathy changes become visible.

\section{REFERENCES}

[1]. Heravian J, Shoeibi N, Azimi A, Yasini S, Moghaddam HO, Yekta AA, EsmailyH. Evaluation of Contrast Sensitivity, Color Vision and Visual Acuity in Patients with and without Diabetes. Iranian J of Ophthalmol 2010;22(3):33-40.

[2]. PK Khosla, D Talwar. Contrast sensitivity changes in background diabetic retionapthy. Can J Ophthalmol 1991;26(1):7-11.

[3]. S Della Sala, G Bertoni et al. Impaired contrast sensitivity in diabetic patients with and without diabetic retinopathy: a new technique for rapid assessment. British Journal of Ophthalmology 1985;(69):136-142.

[4]. Verotti A, Lobefalo L, Petitti Mt et al. Relationship between contrast sensitivity and metabolic control in diabetics with and without diabetic retinopathy. Ann Med 1998;30(4):369-374.

[5]. Dosso AA, J Sommerhalder. Contrast sensitivity in obese dyslipedemic patients with insulin resistance. Arch Ophthalmol 1998(10);1316-20.

[6]. Marco De, Capasso L. Measuring Contrast sensitivity in aretinopathic patients with Insulin Dependent Diabetes Mellitus. Documenta Ophthalmologica. Advances in ophthalmology1997;93(3):199-209.

[7]. Misra S. Saxena S. Kishore P. Bhasker SK. Misra A. Meyer CH. Association of contrast sensitivity with LogMAR visual acuity and glycosylated hemoglobin in non-insulin dependent diabetes mellitus. J Ocul Biol Dis Infor.2010;3(2):60-63. 